feedback on their return and shared new ideas based on their individual experiences. LOROS staff presented relevant topics to Ndi Moyo staff to share palliative care methods and ideologies. Enabling them to identify practical resource needs.

Results LOROS has been able to share knowledge which in turn has improved confidence levels at Ndi Moyo. On a practical level LOROS has provided some basic medical supplies and resources. LOROS has implemented new working processes learnt at Ndi Moyo to improve team working across the hospice. Overall it has enhanced cultural awareness and diversity.

Conclusion The twinning has helped to transform patient care within both organisations through the sharing of skills and knowledge. For LOROS staff the twinning has brought a new level of appreciation for our standards and values.

\section{P-215 TWINNED: SIMILAR TRAITS BUT NOT IDENTICAL}

Peter Gilliam, Dee Curless, Jackie Whiller. Mountbatten, Isle of Wight, UK

\subsection{6/bmjspcare-2018-hospiceabs.240}

Background In 2015, after 20 years in preparation, a hospice in Cyprus opened its doors. As the only hospice on the Island of Cyprus, they were challenging the norms of culture and expectations for palliative and end of life care. Many residents of Cyprus have strong Christian faith which influences their views, a culture where 'life is precious beyond all means'. Many did not understand what a hospice is or does, as there is no word in the Greek language for hospice. Delivering palliative and end of life care to an island community with a shared geographical isolation was familiar to us; given our longevity and stability as an organisation meant that we were uniquely placed to offer the support being sought.

Aims The aim of the Cypriot hospice was to twin with a partner UK hospice to aid in its development, having a shared sense of purpose.

Methods Support included sharing and development of practice and policies along with advice on retail and fundraising activities. Nursing documentation to support the delivery of care and education, training and practical development for staff has been well received. This has been achieved through ongoing exchange visits in both directions.

Results We have seen an increased confidence in the Cypriot hospice staff along with developments and improvements in patient care and practice.

Conclusion What has emerged has been a relationship that is proving to be mutually beneficial to both organisations. It has enabled us to think beyond the realms of our own service, having a greater understanding of the challenges faced in delivering care in a different cultural setting, sharing and learning to improve patient care which is at the heart of what palliative care is all about.

\section{P-216 HOW HOSPICES CAN ADD VALUE TO THE DELIVERY OF LOCAL SUSTAINABILITY AND TRANSFORMATION PLANS (STP)}

${ }^{1}$ Michelle Webb, ${ }^{2}$ Rebecca McConville, 'Kerry Bareham. 'St Barnabas Lincolnshire Hospice, Lincoln, UK; ${ }^{2}$ Lincolnshire STP, Lincoln, UK

10.1136/bmjspcare-2018-hospiceabs.241
Frailty is recognised as a long term condition. However, the syndromes of frailty are often viewed by older people, professionals and society alike as an inevitable part of aging. As a consequence, opportunities are missed to identify people's underlying complex needs including palliative and end of life care needs. This results in people with frailty, often presenting in crisis to health services with conditions that ostensibly appear to have been avoidable.

Lincolnshire has implemented Neighbourhood Team working, underpinned by the principles of the 'House of Care' (HoC) policy: a proactive model of self-care that encourages a move away from the traditional health care delivery model that is single condition specific to one that has a person-centred approach and promotes an integrated model of care. A model that is familiar to providers of specialist palliative and end of life care. Therefore, St Barnabas Lincolnshire Hospice has worked collaboratively with the members of the Neighbourhood Teams to develop a Frailty Pathway to inform the Neighbourhood Teams priorities.

The Frailty Pathway works as a boundary object to facilitate a whole systems approach by the Neighbourhood Teams, thus ensuring people with frailty, wherever they present are able to have their needs recognised, and assessed resulting in personal support and care plans reflecting their outcomes including when appropriate, advance care planning and timely access to palliative and end of life care.

We would like to present our experiences in collaboration with a Neighbourhood Team Lead, to share how hospices can support generalists to improve the health and well-being outcomes for people with multi-comorbidity and frailty, by widening access to palliative and end of life care. Thus enabling people to achieve person-centred outcomes including, preferred place of care, and supporting the STP aspiration of reducing avoidable admissions by $30 \%$.

\section{P-217 COLLABORATIVE NEIGHBOURHOOD TEAM WORKING ENABLING IMPROVED USE OF RESOURCES AND PERSON-CENTRED CARE}

Kerry Bareham, Anita Miller, Karen McCay. St Barnabas Lincolnshire Hospice, Lincoln, UK

10.1136/bmjspcare-2018-hospiceabs.242

The challenge for Lincolnshire reflects that of most of the NHS. In spite of our best efforts our current model does not always deliver the highest quality safe services. Demand for services are increasing, associated with an increasingly aged population with multiple comorbidities and associated complexities. There is a national shortage of appropriately trained and skilled staff, compounded in Lincolnshire with increased challenges in recruitment. We need to look at a way of delivering services that is less reliant on secondary care. We need to stop duplication of services and move away from the traditional reactive model of care the NHS was designed to deliver, to a service model that recognises the biopsychosocial determinants of health and is therefore a more holistic proactive model. Delivered seamlessly by a multi- agency, multidisciplinary team working together to achieve person centred outcomes.

Lincolnshire has adopted a Neighbourhood Team (NHT) model of working that reflects the proactive model of care described by NHS England's House of Care policy - A framework for long term conditions. One of the priorities has been 